\title{
Quantitation of IgG and IgM Response to Recombinant Zika Virus Proteins
}

Imraan Ali, James P. Chambers, Masarrat Ali and Richard C. Tamfu*

Alpha Diagnostics International, Inc., San Antonio Texas, USA

*Corresponding author: Richard C Tamfu, Alpha Diagnostics International, Inc., San Antonio Texas, USA, Tel: 210-561-9515; Fax: 210-561-9544; E-mail: rtamfu@4adi.com

Received date: August 11, 2017; Accepted date: August 30, 2017; Published date: September 05, 2017

Copyright: (C2017 Ali I, et al. This is an open-access article distributed under the terms of the Creative Commons Attribution License, which permits unrestricted use, distribution, and reproduction in any medium, provided the original author and source are credited.

\begin{abstract}
Background: Currently, neither vaccine nor drugs are available for treatment of Zika infection. Vaccines for Flaviviruses have been developed using whole inactivated Yellow Fever virus (YFV) as well as YFV-vectored constructs expressing Dengue Envelop-PrM protein. Thus, Flavivirus proteins show promise as vaccine candidates. Using a rabbit model, the aim of this work was to evaluate the response elicited to recombinant Zika Envelop and NS1 proteins.
\end{abstract}

Methods: His tagged (carboxy terminus) recombinant proteins were expressed in E. coli, purified using Ni-NTA Agarose and imidazole elution, and used to immunize adult New Zealand white male rabbits. Envelop and NS1 antibody ELISAs were developed, and levels of $\lg G$ and $\lg M$ assessed during the course of vaccination.

Results: Zika recombinant Envelop protein elicited a robust IgG response $(4500 \mu \mathrm{g} / \mathrm{ml}$ serum $)$ in contrast to the NS1 protein $(\sim 100 \mu \mathrm{g} / \mathrm{ml}$ serum). Both proteins elicited an IgM response; although, significantly lower, i.e., $\sim 25-50$ $\mu \mathrm{g} / \mathrm{ml}$ serum. E. coli expressed Envelop and NS1 elicited IgG reacted robustly compared with sf9 cell expressed Envelop and NS1 glycosylated homologs. Zika Anti-Envelop lgG cross-reacted with E. coli expressed YFV and Dengue Type I Envelop homologs (approximately 20\% control); whereas, Zika anti-NS1 IgG cross-reacted (approximately $20 \%$ control) with E. coli expressed WNV NS1 homolog.

Discussion and Conclusion: Zika Envelop protein is unique among Flaviviruses; although, parts of it resemble West Nile, Japanese Encephalitis, and Dengue virus homologs. Although some cross-reactivity, i.e., $\sim 25 \% A_{450}$ values was observed, and as with all animal model derived data there are limitations to human extrapolation, data presented here support the potential usefulness of 1) Zika recombinant Envelop protein as a vaccine candidate, and 2) Zika Envelop specific ELISA reagents.

Keywords: Zika; Flavivirus; Recombinant Env and NS1 proteins; Immune response; Protein vaccines

\section{Introduction}

Zika virus is a Flavivirus first discovered in the serum of a rhesus macaque monkey in the Ugandan Zika Forest in 1947, and subsequently in humans [1]. It is transmitted by the Aedes aegypti mosquito [2] which is endemic to the Southern, Eastern, and MidEastern parts of the United States. Due to the large area inhabited by the mosquito [3], and the proximity to South America and the Caribbean where the most recent outbreak originated, it has become a major health concern in the United States [4]. Currently, there has been 5,381 confirmed cases of Zika in the US [5]. The majority of cases observed have been travel-related with the first 14 cases arising by localized transmission in Miami, Florida in July 2016 [5]. Due to the localized nature of infection, the CDC for the first time in its history issued a warning not to travel to an American neighborhood due to an outbreak of an infectious disease [6].

Zika viral infection does not initially give rise to severe symptoms with $80 \%$ of all cases being non-symptomatic, and the remaining $20 \%$ giving rise to minor flu-like symptoms [7]. Currently, no vaccine or anti-viral therapeutic agents exist for treatment of Zika infection [7].
The major concern for Zika viral infection is the increase in microcephaly observed in pregnant women in mosquito infected areas prompting the CDC to conclude Zika virus as being the causative agent [8]. Microcephaly is a lifelong condition associated with much smaller head size-giving rise to symptoms ranging from seizures, developmental delay, cognitive deficit with movement/balance, feeding, hearing, and vision problems, and in severe cases death [9]. Additionally, Guillain-Barré Syndrome (GBS) is a peripheral nervous system disorder increasingly observed in individuals in areas where Zika virus is prevalent [9]. Although the greater majority of GBS individuals recover from the most severe cases, some individuals experience long-term symptoms [8].

\section{Materials and Methods}

\section{Recombinant protein expression and purification}

cDNA for Zika virus envelop protein corresponding to amino acids 1-251 (Accession\# AHL43464.1), and cDNA for Zika virus NS1 protein corresponding to amino acids 795-1148 (Accession\# BAP47441.1) were optimized and synthesized for expression in E.coli. Each respective cDNA was sub-cloned into pET28A vector using NcoI and XhoI restriction sites. Following transformation of BL21 (DE3) 
cells with each construct, respective colonies were screened for recombinant protein expression. Briefly, cells were grown in LBKanamycin at $37^{\circ} \mathrm{C}$ in a shaker $(150 \mathrm{rpm})$ to $\mathrm{OD}_{600}$ of $0.5-0.6$ followed by induction with $0.5 \mathrm{mM}$ IPTG for recombinant protein expression for an additional 3 hours. Cells were lysed in $50 \mathrm{mM}$ Tris- $\mathrm{HCl}, \mathrm{pH} 8.0$ buffer containing $0.2 \mathrm{mg} / \mathrm{ml}$ lysozyme, $0.5 \mathrm{mM}$ EDTA, $1 \mathrm{mM}$ PMSF, 5 $\mathrm{mM} \beta$-mercaptoethanol and $250 \mathrm{mM} \mathrm{NaCl}$ on ice. Lysates were sonicated 3 times ( 30 seconds) on ice (60\% full power setting). Whole cell lysate material was centrifuged at $10,000 \mathrm{rpm}$ for 1 hour using a GS3 rotor to pellet inclusion bodies. Inclusion bodies were washed twice in the above buffer, and inclusion body proteins were extracted in buffer containing $8 \mathrm{M}$ urea. His-tagged extracted proteins were applied to a Ni-NTA Agarose column, and eluted with buffer containing $0.25 \mathrm{M}$ imidazole. Protein was quantitated using the BioRad dye-binding assay [10], and BSA as standard. Protein purity was assessed by SDS-PAGE, and proteomic analysis (see below).

\section{SDS-PAGE and proteomic analysis of recombinant proteins}

Electrophoresis was carried out under denaturing conditions according to the method of Laemmli [11]. Recombinant Envelop and NS1 protein (1.6 and $1.4 \mu \mathrm{g}$, respectively) were heated for 5 minutes at $95^{\circ} \mathrm{C}$ in the presence of SDS and $\beta$-mercaptoethanol, and applied to $10 \%$ BIS-Tris gels (Life Sciences Technology, Inc., Hewlett, NY). Electrophoresis was carried out at constant voltage (80V) for 1.5-2.0 hours at room temperature. Gels were stained with Coomassie Blue. The gel bands of interest were reduced in situ with TCEP [tris(2carboxyethyl)phosphine] and alkylated in the dark with iodoacetamide prior to treatment with trypsin (Promega). The digests were analyzed by capillary HPLC-electrospray ionization tandem mass spectrometry (HPLC-ESI-MS/MS) on a Thermo Fisher Orbitrap Fusion Lumos mass spectrometer fitted with a New Objective PicoView 550 nanospray interface. On-line HPLC separation of the digests was accomplished with an RSLC NANO HPLC system (Thermo Fisher/Dionex). Precursor ions were acquired in the orbitrap from m/z 300-m/z 1500 at 120,000 mass resolutions (m/z 200); data-dependent higher-energy collision dissociation (HCD) spectra were acquired at the same time in the linear trap using the "top speed" option. Mascot (Matrix Science) was used to search the spectra against the virus subset of the UniProt database (downloaded on 4/11/2017) concatenated to a database of common protein contaminants. Cysteine carbamidomethylation was set as a fixed modification and methionine oxidation, deamidation of glutamine and asparagine, and acetylation of the protein $\mathrm{N}$-terminus were considered as variable modifications; trypsin was specified as the proteolytic enzyme, with one missed cleavage allowed. Subset searching of the identified proteins was achieved by X! Tandem, crosscorrelation with Mascot results and determination of protein and peptide identity probabilities was accomplished by Scaffold (Proteome Software). The thresholds for acceptance of peptide and protein assignments in Scaffold were 95\% and 99\%, respectively, two peptides minimum per protein (peptide and protein $\mathrm{FDR}<0.1 \%$ ). Mass spectrometry analyses were conducted in the Mass Spectrometry Core Laboratory of The Department of Biochemistry, The University of Texas Health Science Center at San Antonio.

\section{Animals}

Adult, New Zealand White male (5-6 lbs.) rabbits were used for antibody production in a USDA sanctioned facility with approved IACUC protocols.

\section{Immunization protocol}

Rabbits (one for each antigen) were immunized by subcutaneous injection (3-4 sites) of recombinant Zika protein antigens: Envelop protein $50 \mu \mathrm{l}(135 \mu \mathrm{g}$ protein/ml) and NS1 protein, $50 \mu \mathrm{l}(130 \mu \mathrm{g}$ protein $/ \mathrm{ml}$ ). Due to very low antibody titers following 5 injections, NS1 dosage was doubled, i.e., $260 \mu \mathrm{g} / 50 \mu \mathrm{l}$ for injections 6 and 7. A pre-immune bleed was collected prior to immunization (Day 0). Immunization was initiated on Day 1 , and subsequently performed at $2,4,6,8,10$, and 12 weeks. Bleeds were collected at $3,7,9,14,16$, and 17 weeks, respectively. Serum was separated at room temperature by centrifugation (15 minutes at $3000 \mathrm{rpm}$ ), and stored at $-20^{\circ} \mathrm{C}$ until used.

\section{ELISA plate coating}

ELISA plates were prepared by coating overnight at $4^{\circ} \mathrm{C}$. For Envelop protein $\operatorname{IgG}$ and $\operatorname{IgM}$ detection, antigen was coated at a concentration of $0.5 \mu \mathrm{g} / \mathrm{ml}$ in PBS. For NS1 IgG and IgM detection, NS1 antigen was coated at concentrations of 0.1 and $0.5 \mu \mathrm{g} / \mathrm{ml}$, respectively, in PBS. For cross-reactivity assessment (IgG only), Flavivirus Envelop and NS1 related recombinant proteins were coated at $0.2 \mu \mathrm{g} / \mathrm{ml}$. Plates were washed with $1 \mathrm{X}$ wash buffer 3 times. Stablecoat reagent $(100 \mu \mathrm{l})$ was added to each well, incubated at room temperature for 1 hour after which time wells were aspirated using an ELISA wash apparatus, and subsequently dried at $30^{\circ} \mathrm{C}$ for 3 hours.

\section{ELISA}

Zika Envelop and NS1 ELISAs were carried out as follows: serum $(100 \mu \mathrm{l})$ was added to each well and incubated for 1 hour. Wells were washed ( 3 times) with wash buffer followed by addition of $100 \mu \mathrm{l}$ horse radish peroxidase (HRP) conjugated immunoglobulins, i.e., Goat antiRabbit IgG or Goat ant-Rabbit IgM (Alpha Diagnostic International, San Antonio, TX), and incubated for 30 minutes at room temperature.

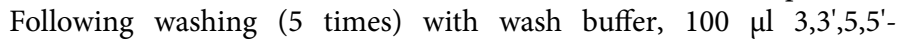
Tetramethylbenzidine (TMB) substrate was added to each well, incubated for 15 minutes at room temperature, and reactions were terminated by addition of $100 \mu \mathrm{l}$ 'Stop' solution. Absorbance at $450 \mathrm{~nm}$ was determined using an ELISA Plate reader (MPR-2100, Awareness Technologies, Westport, CT).

\section{Generation of standard curve and quantitation of ELISA IgG/IgM}

Titer strips were generated as follows: Rabbit IgG and IgM (Equitech Bio, Kerville, TX) was coated at specific concentrations, i.e., 3, 10, 30, and $100 \mathrm{ng}$, respectively. HRP-conjugate $(100 \mu \mathrm{l}$ Goat-anti Rabbit IgG or Goat-anti Rabbit IgM) was added to strips at appropriate dilutions (IgG, 1:200; IgM, 1:100) for 30 minutes. Following washing (5 times), $100 \mu \mathrm{TMB}$ substrate was added to each well, incubated for 15 minutes at room temperature, and absorbance at $450 \mathrm{~nm}$ determined following addition of 'Stop' solution. Absorbance for standards was linear from 3 to $100 \mathrm{ng}$. ELISA absorbance values were all quantitated within this linear range by appropriate dilution. Concentration was determined by multiplication of respective extrapolated $\mathrm{A}_{450}$ values by the dilution necessary to achieve $A_{450}$ values within the standard linear range.

\section{Results}

SDS-PAGE analysis of expressed Envelop and NS1 recombinant proteins used in this study is shown in Figure 1. 
SDS-PAGE analysis of Zika Envelop and NS1 recombinant proteins
A.

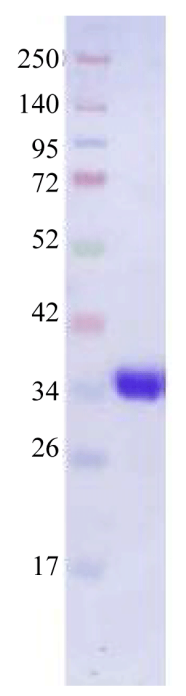

B.

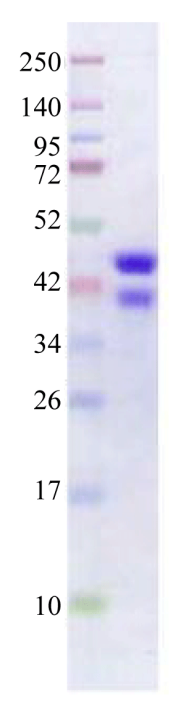

Figure 1: SDS-PAGE and sequencing analysis was carried out as previously described under 'Materials and Methods'. Frame A: Zika Envelope. Frame B: Zika NS1.
Envelop recombinant protein migrated as a single electrophoretic band of approximately $35 \mathrm{kDa}$ (Frame A); whereas, NS1 recombinant protein migrated as 2 electrophoretic bands of approximately 40 (arising from truncation) and $45 \mathrm{kDa}$, respectively (Frame B). The identity of both recombinant proteins used in this study was validated by Mass Spectrometry (data not shown). Immunization with recombinant Envelop and NS1 proteins elicited high raw absorbance values starting about day 21 , increasing thereafter to approximately day 110 , and decreasing slightly thereafter out to day 120 (Figure 2A). Shown in the inset (Figure 2B) is a representative standard curve used to quantitate, i.e., titer the respective antigen response indicating increasing levels of Envelop protein elicited IgG from day 20 out to day $110(4,500 \mu \mathrm{g} / \mathrm{ml})$. The IgG response to NS1 recombinant protein also increased in similar fashion, but appears at background level due to scaling (Figure 2B) increasing steadily from day 20 out to approximately day 90 post-immunization, i.e., $\sim 100 \mu \mathrm{g} / \mathrm{ml}$ (data not shown).

In contrast to the Zika recombinant antigen IgG response, raw absorbance values for IgM appear biphasic in nature with maxima for recombinant Envelop protein elicited at approximately day 20 and 110 post-immunization (Figure 3A); whereas, NS1 elicited IgM raw absorbance values indicated maxima at approximately day 50 and 110 post-immunization (Figure 3B). Quantitation of the elicited IgM response was lower but gradual for NS1, i.e., $\sim 25 \mu \mathrm{g} / \mathrm{ml}$ at 110 days post-immunization compared to that observed for Envelop recombinant protein $(\sim 55 \mu \mathrm{g} / \mathrm{ml}$ at 20 and 110 days postimmunization).

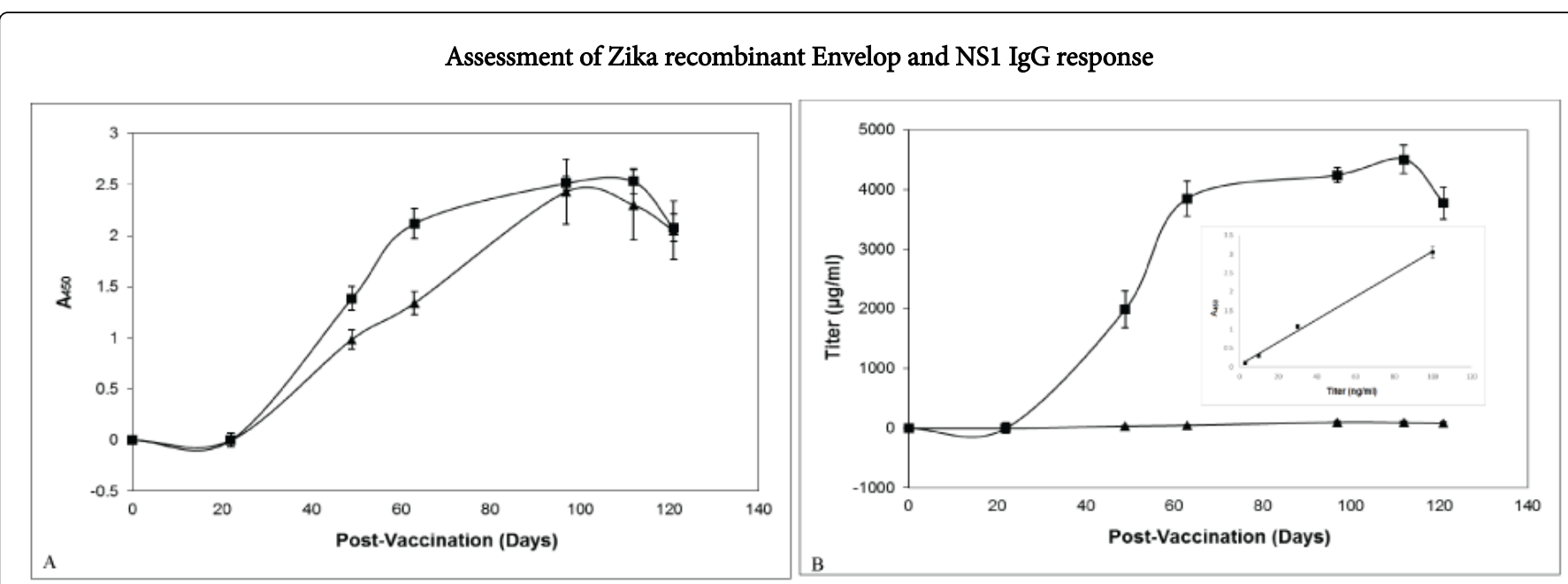

Figure 2: NS1 and Envelop recombinant protein elicited IgG for different bleeds was determined as previously described under 'Materials and Methods'. Frame A: Anti-serum raw absorbance (mean value of three determinations) values (closed squares=Envelop protein, closed triangles = NS1 protein). A Dilution factor of 1:60,000 and 1:1,500 for Envelop and NS1 recombinant protein was used, respectively. Frame B: Envelop (closed squares) and NS1 (closed triangles) IgG titers (mean value of 3 determinations) as a function of post-vaccination time. The titer of elicited IgG was achieved by extrapolation to a standard curve. Inset: Rabbit IgG Standard Curve. A rabbit IgG titer strip was prepared as described under 'Materials and Methods', and standard curve derived (mean value of 3 determinations, $y=33.02 \times-5.82$ ).

Zika Envelop recombinant protein (expressed in E. coli) antiserum, i.e., IgG was evaluated for cross-reactivity with recombinant Envelop and NS1 homologs from related Flaviviruses. While we observed no cross-reactivity with West Nile virus (WNV), Chikungunya virus (CV), and Japanese Encephalitis virus (JEV) homologs, we did observe approximately $20 \%$ control $\mathrm{A}_{450}$ values (E. coli expressed Zika
Envelope elicited response) cross-reactivity with Yellow Fever (YFV) and Dengue Type 1 virus homologs. Antiserum obtained from rabbits vaccinated with $E$. coli Zika NS1 recombinant protein exhibited approximately $20 \%$ control $\mathrm{A}_{450}$ values (E. coli expressed Zika NS1 elicited response) cross-reactivity with WNV recombinant NS1 homolog (Figure 4). 


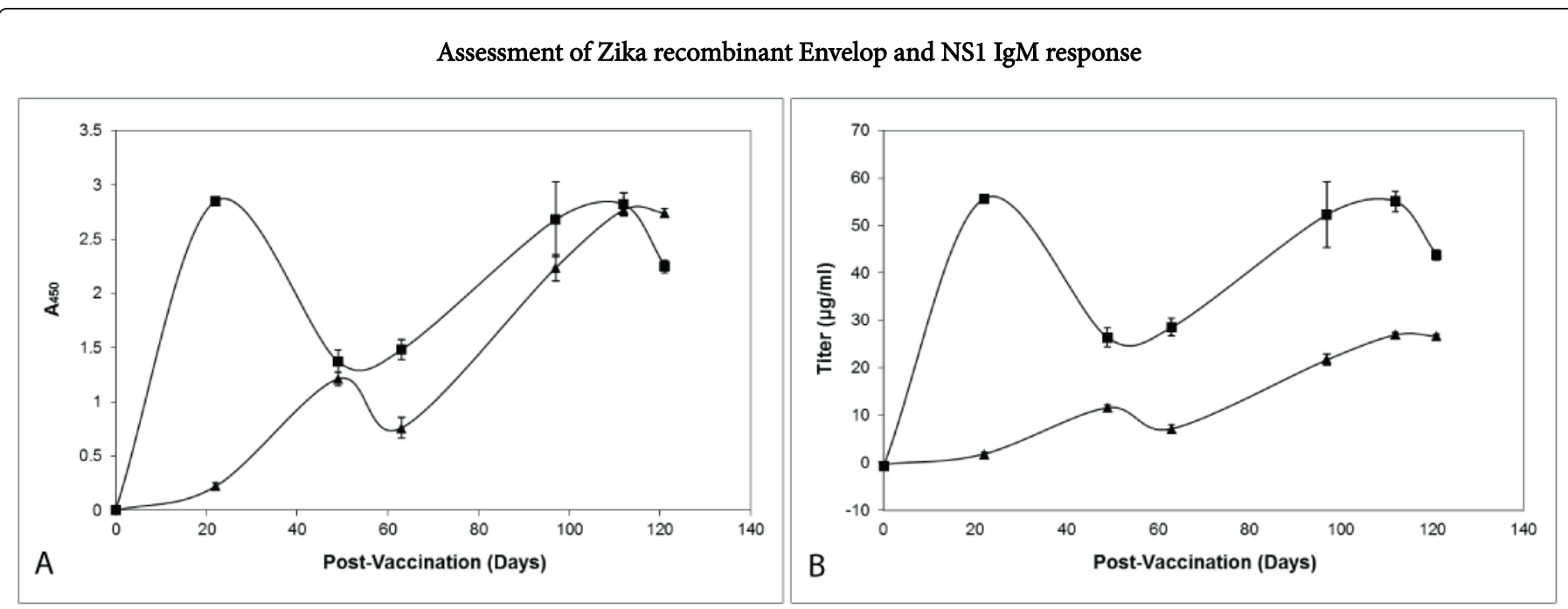

Figure 3: NS1 and Envelop recombinant protein elicited IgM for different bleeds was determined as previously described under 'Materials and Methods'. Frame A: Anti-serum raw absorbance (mean value of three determinations) values (closed squares=Envelop protein; closed triangles=NS1 protein). A Dilution factor of 1:600 and 1:300 for Envelop and NS1 recombinant protein was used, respectively. Frame B: Envelop (closed squares) and NS1 (closed triangles) IgM titers (mean value of 3 determinations) as a function of post-vaccination time. The titer of elicited IgM was achieved by extrapolation to a standard curve essentially identical to that shown above. A rabbit IgM titer strip was prepared as described under 'Materials and Methods' and standard curve generated (mean value of 3 determinations, $y=33.03 \times-1.35$, data not shown).

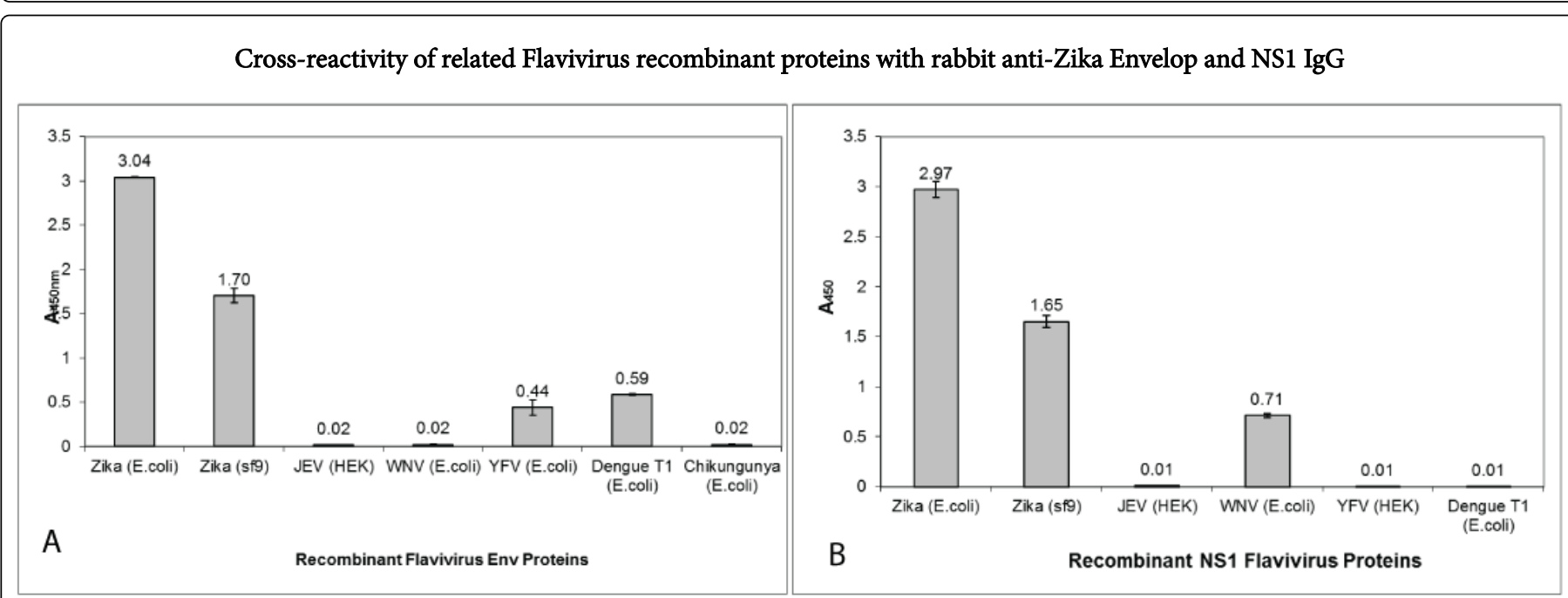

Figure 4: ELISA plate preparation for cross-reactivity assessment was carried out as described under 'Materials and Methods'. Error bars represent 4 replicate determinations at a dilution of 1:5000 serum with all proteins coated at $0.2 \mu \mathrm{g} / \mathrm{ml}$. Frame A: Flavirirus recombinant Envelop proteins. Frame B: Flavivirus recombinant NS1 proteins. The mean raw absorbance value is shown above each respective bar.

\section{Discussion}

Zika recombinant Envelop protein elicited a robust IgG response starting approximately 3 weeks post-immunization 'up' to 'out' continuing up to 110 days $(\sim 4500 \mu \mathrm{g} / \mathrm{ml})$ consistent with an expected 'adaptive' albeit continuous response following 5 subsequent injections, i.e., roughly 4 months after the initial injection. In contrast, IgG elicited in response to Zika recombinant NS1 protein albeit lower was significant $(\sim 100 \mu \mathrm{g} / \mathrm{ml}$ at day 90 post immunization). Both Envelop and NS1 recombinant protein elicited IgM levels were observed to be lower in comparison ( 2 maxima of approximately $50 \mu \mathrm{g} / \mathrm{ml}$ at 20 and 110 days, and 10 and $25 \mu \mathrm{g} / \mathrm{ml}$ at 50 and 110 days post-immunization, respectively) than that observed for IgG. One possible explanation for the much lower IgM titers is inhibition or cross-reactivity, i.e., competition of immune reagents elicited to similar/identical epitopes. Future experiments involving selective precipitation of serum IgG and subsequent IgM quantitation could shed further light on this issue. If IgG competitively blocks IgM binding to the target antigen, detectable 
IgM levels should increase in its absence. It is also noteworthy that a portion of the NS1 recombinant protein was truncated as determined by mass spectroscopy and could contribute to the lower NS1 elicited IgG and IgM responses. NS1 elicited antibodies could be used to discriminate animals previously vaccinated with subunit or killed vaccine which does not elicit antibodies toward NS1 protein from those with active Zika viral infection.

Flaviviruses (Zika, Dengue, West Nile, Japanese Encephalitis, and Yellow Fever) have similar genetic makeup, and the primary antigenic proteins (Envelop, prM, and NS1) share $50-70 \%$ protein sequence homology across the group with large regions in Envelop and NS1 proteins more conserved within the Flavivirus family than all others. However, only approximately $20 \%$ cross-reactivity of rabbit anti-Zika Envelop and NS1 antibodies with related Flavivirus recombinant homologs was observed. Although, Zika Envelop and NS1 proteins are glycosylated and recombinant proteins derived in this study are not, antibodies from rabbits vaccinated with $E$. coli derived recombinant Envelop and NS1 proteins reacted robustly with glycosylated Flavivirus Envelop and NS1 homologs.

Currently a number of vaccines are in development using recombinant proteins. Although recombinant protein vaccines potentially can be less effective due to being less immunogenic as natural pathogen proteins, i.e., eliciting a less robust Tc response, such an approach offers some advantage over live, attenuated or inactivated vaccines, e.g., stability, no need to use whole organisms, genetic manipulation to increase immunogenicity, and no chance of mutation thus reversing attenuation as in live vaccines. Attenuated recombinant Yellow Fever virus containing the Zika Envelop gene is currently being produced by Sanofi Pasteur (Lyon France). Additionally, Bharat Biotech (Hyderabad, India) and Protein Sciences Corporation (Meriden, CT) are using viral surface antigen expression to induce immune responses [12].

As with all animal model derived data, there are serious limitations to human extrapolation, e.g., vaccination of rabbits was carried out using a Mycobacterium adjuvant that is highly antigenic persisting much longer than other adjuvants [13]. However, Mycobacterium adjuvants are banned for human use by the FDA due to toxicity, and can only be injected subcutaneously or intraperitoneally in animals due to complications that can arise via other routes. Alternatively, aluminum salt adjuvants are available for human vaccine development but could lead to lower antibody titers, as they are not as persistent or antigenic. Additionally, in the rabbit model described here, booster immunizations were given every 2 weeks in contrast to that used for human vaccination in which booster immunizations are typically given several months to a year apart.

\section{Conclusion}

The Envelop protein is a major viral surface protein involved in various steps of the Zika virus life cycle. IgG titers produced in response to both Zika Envelop and NS1 recombinant proteins increased with each subsequent injection, i.e., 3 through 7; the peak NS1 IgG titer being roughly 45 -fold less than that observed for Envelop recombinant protein. IgM levels elicited by both Envelop and NS1 recombinant proteins were lower, i.e., approximately 82 - and 180 -fold less, respectively, than the IgG response to the Envelop recombinant protein. Early sequence and structural comparisons of the Zika Envelop protein with that of other Flaviviruses suggest that overall the Zika Envelop protein is unique among Flaviviruses; although, parts of it resemble its homologs in West Nile, Japanese Encephalitis, and Dengue viruses $[14,15]$. This is consistent with the small amount of cross-reactivity of rabbit anti-Zika Envelop elicited antibodies with related Flavivirus recombinant protein homologs. It remains to be determined if the antibodies produced are effective at limiting Zika virus transmission. Data reported here support the potential of 1 ) the Zika Envelop protein as a vaccine candidate [16,17], 2) usefulness of the NS1 response in discriminating prior vaccine exposure from active viral infection, and 3) usefulness of Zika specific ELISA reagents.

\section{Acknowledgements}

We dedicate this work to the memory of our dear colleague Dr. Masarrat Ali who passed away unexpectedly, April 21, 2017. We gratefully acknowledge Alpha Diagnostics International, Inc. for financial support of this work. The expert technical assistance of Sammy Pardo and Dana Molleur of the Mass Spectrometry Core Laboratory at the University of Texas Health Science Center at San Antonio is gratefully acknowledged.

\section{References}

1. Sikka V, Chattu VK, Popli RK, Galwankar SC, Kelkar D, et al. (2016) The emergence of zika virus as a global health security threat: A review and a consensus statement of the INDUSEM Joint working Group (JWG). J Global Infect Dis 8: 3-15

2. Malone RW, Homan J, Callahan MV, Glasspool-Malone J, Damodaran L, et al. (2016) Zika Virus: Medical Countermeasure Development Challenges. PLoS Negl Trop Dis 10: e0004530.

3. Gubler DJ (2011) Dengue, Urbanization and Globalization: The Unholy Trinity of the 21st Century. Trop Med Health 39: 3-11.

4. World Health Organization Zika virus infection (2016) Global update on epidemiology and potentially associated clinical manifestations. Wkly Epidemiology Rec 91:73-81.

5. Centers for Disease Control and Prevention (2017) Zika Virus.

6. Centers for Disease Control and Prevention (2016) Additional area of active Zika transmission identified in Miami Beach.

7. Centers for Disease Control and Prevention (2016). Symptoms, Testing, and Treatment.

8. Centers for Disease Control and Prevention (2016) CDC Concludes Zika Causes Microcephaly and Other Birth Defects.

9. Rasmussen SA, Jamieson DJ, Honein MA, Petersen LR (2016) Zika virus and birth defects-reviewing the evidence for causality. N Eng J Med 374: 1981-1987.

10. Bradford, $M$ (1976) A rapid and sensitive method for the quantitation of microgram quantities of protein utilizing the principle of protein dye binding. Analytical Biochemistry 72: 248-254.

11. Laemmli UK (1970) Cleavage of structural proteins during the assembly of the head of bacteriophage T4. Nature 227: 680-685.

12. Waltz E (2016) GM mosquitoes fire first salvo against Zika virus. Nature Biotechnology 34: 221-222.

13. Harrison WT (1935) Some Observations on the Use of Alum Precipitated Diphtheria Toxoid. Am J Public Health Nations Health 25: 298-300.

14. Cox BD, Stanton RA, and Schinazi RF (2015) Predicting Zika virus structural biology. Challenges and opportunities for intervention. Antivir Chem Chemother 24: 118-126.

15. Kostyuchenko VA, Lim E, Zhang S, Fibriansah G, Ng T-S, et al. (2016) Structure of the thermally stable Zika virus. Nature 533: 425-428.

16. Coller BA, Clements DE, Bett AJ, Sagar SL, Ter Meulen JH (2011) The development of recombinant subunit Envelop-based vaccines to protect against dengue virus induced disease. Vaccine 29: 7267-7275.

17. Miller N (2010) Recent progress in dengue vaccine research and development. Curr Opin Mol Ther 12: 31-38. 